\title{
Oxidative Stress Indices and Inflammatory Responses in Female Wistar Rats Administered Acetaminophen, Ampicillin/Cloxacillin and Co-Trimoxazole
}

\author{
Oyewo $\mathrm{EB}^{1 *}$, Ajayi J0 ${ }^{1}$, Adekunle $\mathrm{AS}^{1}$ and 0 so $\mathrm{JB}^{2}$ \\ ${ }^{1}$ Department of Biochemistry, Faculty of Basic Medical Sciences, LadokeAkintola University of Technology, Ogbomoso, Oyo \\ State, Nigeria
}

${ }^{2}$ Department of Biochemistry, Faculty of Biosciences, McPherson University, Ogbomoso, Oyo State, Nigeria

*Corresponding author: Emmanuel Bukoye Oyewo, Department of Biochemistry, Faculty of Basic Medical Sciences, LadokeAkintola University of Technology, Ogbomoso, Oyo State, Nigeria

\section{ARTICLE INFO}

Received: 㹃 August 12, 2021

Published: 幽 August 23, 2021

Citation: Oyewo EB, Ajayi JO, Adekunle AS, Oso JB. Oxidative Stress Indices and Inflammatory Responses in Female Wistar Rats Administered Acetaminophen, Ampicillin/Cloxacillin and Co-Trimoxazole. Biomed J Sci \& Tech Res 38(2)-2021. BJSTR. MS.ID.006135.

Keywords: Prescription Drugs; Acetaminophen; Co-trimoxazole; Ampicillin/ cloxacillin; Oxidative stress; Inflammatory Responses; Vascular related disorders

\begin{abstract}
Prescription drugs are frequently purchased from Pharmacy outlets based on selfmedication and often, combined to achieve therapeutic objectives that may trigger drug interactions that have not been fully elucidated. Therefore, this study evaluated the effects of concomitant administration of acetaminophen, ampicillin/cloxacillin and cotrimoxazoleon oxidative and inflammatory responses. Seventy female Wistar rats were assigned into seven groups of ten animals each. Therapeutic doses at $100 \mathrm{mg} / \mathrm{kg}$ body weight of co-trimoxazole, and $105 \mathrm{mg} / \mathrm{kg}$ body weight each of ampicillin/cloxacillin and acetaminophen orally once daily for four weeks. Group A served as the control, group B was administered ampicillin/cloxacillin only, group C was administered co-trimoxazole only, group D was administered acetaminophen only, group E was administered acetaminophen and ampicillin/cloxaxillin, group $\mathrm{F}$ was administered co-trimoxazole and acetaminophen, and group G was administered acetaminophen, ampicillin/ cloxacillin, and co-trimoxazole. Oxidative status was determined in the serum and homogenates of liver and brain by the levels of total thiol, reduced Glutathione (GSH), Oxidized-Low Density Lipoprotein Cholesterol (Ox-LDL-C). Inflammatory response was assessed by measuring the serum levels of Interleukin-1 $\beta$ (IL-1 $\beta$ ), Nitric Oxide (NO), Monocyte Chemoatracttant Protein (MCP-1), Vascular Adhesion Molecule (VCAM-1) and Total Immunoglobulin G (IgG). Body weights and organ to body weight ratios across the groups were insignificantly different $(p>0.05)$. Serum total thiol level increased $(p<0.05)$ in B and D compared to A,C, E, F and G. Liver and brain total thiol levels were insignificant changed ( $p>0.05)$ across the groups compared to control but increased $(p<0.05)$ in liver of G compared to D and brain of B and D compared to C. Serum GSH levels were insignificantly different ( $p>0.05$ ) across the groups, while liver GSH in G decreased $(p<0.05)$ compared to A, B, C and D and brain GSH level in F was elevated $(p<0.05)$ compared to A,B,C,D and E. Serum Ox-LDL-C concentrations were elevated significantly $(p<0.05)$ in F and G compared to A and D. Serum levels of IL-1 3, NO, MCP-1,VCAM-1 and $\operatorname{IgG}$ were insignificant changed $(p>0.05)$ across the groups, but there was decrease $(\mathrm{p}<0.05)$ in VCAM-1 in G compared to B. Histological examination indicated varying degrees of vacuolation, fenestration and occlusion in liver, and neurodegeneration in brain of groups administered the drugs. This study showed that the concomitant use of acetaminophen, ampicillin/cloxacillin and co-trimoxazole caused oxidative stress that has a weak link to vascular related disorders. Therefore, awareness of the health implications associated with misuse of these drugs should be emphasized.
\end{abstract}




\section{Introduction}

Drug is any chemical substance which when taken into the body interacts with tissues to modify the biochemical process of the body. A drug is abused when consumed in amounts or with methods which are harmful to the body [1]. Conventionally, self-medication involves the use of drugs, herbs or other home remedies on an individual's persuasion without prescription and it is a frequent practice throughout the world with a high prevalence rate in developing countries [2-5]. Analgesics are the most frequently selfmedicated over-the-counter drugs while antibiotics are considered as prescription-only medicines [6], however, a number of them can be purchased from pharmacy outlets without a prescription. This is especially true with co-trimoxazole and the penicillins (ampicillin/ cloxacillin). The use of analgesics are known to be influenced by stress levels and a large percentage of users take analgesics before the commencement of the day's work [7], in anticipation of pain or to offset any form of body pain. For instance, acetaminophen which is a ubiquitous analgesic is commonly used for relief at the slightest feeling of aches, headaches, symptoms of flu and pains $[8,9]$. While acetaminophen is described as relatively nontoxic when administered in therapeutic doses [10], it causes toxicity when taken in a single or repeated high dose, or after chronic ingestion.

Similarly, the inappropriate uses of antibiotics for common colds or upper respiratory infections and disorders that are largely self-limiting have been shown by several studies [11]. Cotrimoxazole also known as trimethoprim-sulfamethoxazole, was introduced in 1968 as a broad-spectrum antimicrobial agent. It is a well-tolerated, widely available, off patent, and inexpensive unique compound that combines broad antimicrobial activity with immunomodulatory properties. Clinically, co-trimoxazole is used majorly for the treatment of urinary tract infections and also infections of respiratory and gastrointestinal tracts. Ampicillin/ cloxacillin is a mere analogue of the penicillins [12]. It is used majorly to treat infections of the middle ear, sinuses, bladder, kidney, and uncomplicated gonorrhea. Furthermore, ampicillin/ cloxacillin is also used intravenously to treat meningitis and other serious infections while cloxacillin is used against staphylococci that produce $\beta$-lactamase [13]. Due to their high level of safety, the penicillins are the most widely prescribed antibiotic in the world against both gram positive and gram negative cocci [12]. Since they were the first class to be developed, it is believed that the familiarity of the population to this class of antibiotics could contribute to their misuse and abuse. Also, because of it low cost ampicillin/cloxacillin is the most commonly purchased antibiotic without prescription [14].

Available lines of evidence have revealed that the bactericidal effect of antibiotics is often associated to production of Reactive Oxygen Species (ROS) [15,16], and that drugs such as anti- inflammatory and analgesics could induce oxidative stress [17]. Consequently, the progression of oxidative stress is linked to inflammation in a complex feedback cycle such that reactive oxygen species can trigger transcription factors that up-regulate the expression of pro-inflammatory cytokines, pro-oxidant and antioxidants [18]. Previous studies have indicated the common coadministration of analgesics and antibiotics for the management of pain while treating various infections [19]. In addition to this, low cost and easy accessibility to some analgesics (acetaminophen, salicylic acid etc) and some antibiotics (co-trimoxazole and ampicillin/ cloxacillin) have escalated their frequent usage prophylactically without prescription [20]. Although, the combination of drugs is often essential to obtain a therapeutic objective or to treat different diseases existing at the same time, but there could be the risk of multiple drug interactions. The outcome of concomitant administration of two or more drugs is usually followed by several therapeutic implications ranging from opposition, alteration, synergism, and potentiating, as well as physical and chemical antagonism, thereby raising a lot of concerns about potential drug interaction [21]. Therefore, this study evaluated the effects of concomitant administration of acetaminophen, ampicillin/ cloxacillin and co-trimoxazole on antioxidant and immunological status in Wistar rats.

\section{Materials and Methods}

\section{Materials}

A. Experimental Animals: Seventy apparently healthy male Wistar rats with weights of 112-128 g were obtained from the animal house facility, Ladoke Akintola University of Technology, Ogbomoso, Oyo State, Nigeria.

B. Drugs: Acetaminophen was a product of Emzor Pharmaceutical industries limited, Plot 3C, Block A, Aswani Market Road, Off Oshodi-Apapa Express Way, Isolo, Lagos, Ampicillin/ cloxacillin was a product of Meidrich Limited. 12th mile, Old Madras Road, Virgonagar, Bangalore- 560 049, India and Cotrimoxazole was a product of GlaxosmithKline S.A.E, El Salem City, 11491, Cairo, A.R.E Under License of the GlaxosmithKline Group of Companies.

C. Reagents Kits and Chemicals: Quantitative assay kits for GSH, total immunoglobulin G are products of Fortress Diagnostic Laboratory, Unit 2C, Antrim Technology Park, Belfast Road, Antrim, Northern Ireland, United Kingdom, interleukin$1 \mathrm{~b}$, nitric oxide, vascular adhesion molecule- 1 are products of RayBio Technology inc USA, while oxidized low-density lipoprotein cholesterol and monocyte chemoattractant protein are products of Bioassay Technology Laboratory, Junjiang Inter Bldg 218 Ningguo Rd. Yangpu Dist. Shangai, China. 


\section{Methods}

Experimental Design: The rats were randomly divided into seven groups of ten rats per group as illustrated:

A. Group A: All rats were allowed free access to standard pellet and water

B. Group B: Was administered Ampicillin/cloxacillin only

C. Group C: Was administered Co-trimoxazole only

D. Group D: Was administered Acetaminophen only

E. Group E: Was administered Acetaminophen and Ampicillin/ cloxacillin

F. Group F: Was administered Acetaminophen and Cotrimoxazole

G. Group G: Was administered Acetaminophen, Ampicillin/ cloxacillin, and Co-trimoxazole

Conversion from human dose to animal dose was done using the equation by Reagan-Shaw et al. [22]. The administration was done once daily at 4:00 pm using $1 \mathrm{ml}$ cannula syringe. $105 \mathrm{mg} / \mathrm{kg}$ body weight each of acetaminophen and ampicillin/cloxacillin was orally administered while $100 \mathrm{mg} / \mathrm{kg}$ body weight of co-trimoxazole was orally administered. The administration lasted for four weeks, after which all the animals were sacrificed.

\section{Collection of Samples}

Serum: After four weeks of administration, the animals were fasted overnight and anesthetized using diethyl ether. The chest region was quickly opened and blood was drawn by puncturing the heart using a new syringe for each animal. Serum was prepared from each drawn blood. The blood samples were centrifuged at $4000 \mathrm{rpm}$ for 10 minutes. The serum was carefully decanted into sample bottles using a micropipette, labeled and preserved in the refrigerator below $4{ }^{\circ} \mathrm{C}$ immediately for further analysis.

Tissue Homogenates: Organs of interest, the liver and brain were harvested immediately, cleansed of blood and rinsed with normal saline solution and the weight recorded. The organs were minced and a small part was fixed. The organs were fixed in $10 \%$ formal saline solution for histopathology examination.0.2 $\mathrm{g}$ of the liver and brain, were homogenized separately in $0.25 \mathrm{M}$ sucrose solution in the ratio 1:4 using a laboratory homogenizer and centrifuged at $4000 \mathrm{rpm}$ for 10 minutes, after which the supernatants were collected and placed in Eppendorf tubes and stored at $4{ }^{\circ} \mathrm{C}$ for further analysis.

\section{Biochemical Assay Methods}

Oxidative Status: The serum and tissues total thiol content were determined using the method of Sedlak and Lindsay [23].
The method of Ellman was used in measuring the concentration of reduced glutathione [24]. Oxidized-Low Density Lipoprotein Cholesterol (Ox-LDL-C) concentration was determined by ELISA as described in the kit manual.

Inflammatory Responses: Nitric Oxide (NO) was determined using spectrophotometric method as described by Schmidt et al. by quantifying the accumulation of its stable degradation products, nitrate, and nitrite [25]. Based on the sandwich immunoassay principle interleukin-1 beta protein, Monocyte Chemoattractant Protein-1 (MCP-1) and vascular cell adhesion molecule-1 analyses was done by ELISA method. Total immunoglobulin G (IgG) content was determined based on immunoturbidimetric method as described in the kit manual.

\section{Histological Assessment}

Histological procedures were done according to the method of Avwioro [26].

\section{Statistical Analysis}

This research work was a Completely Randomised Design (CRD). Results were expressed as mean \pm standard deviation (SD). Data generated were subjected to one way Analysis of Variance (ANOVA), after which Tukey Test was conducted in other to identify the variation within the treatment group. P-value $<0.05$ was regarded as statistically significant and denoted by alphabets.

\section{Results}

\section{Effect of Acetaminophen, Ampicillin/Cloxacillin and Co- Trimoxazole on Oxidative Status}

The results in Table 1 showed a significant increase $(\mathrm{p}<0.05)$ in serum total thiol level in group B and D (ampicillin/cloxacillin and acetaminophen) when compared to the control (group A). Although, there were no significant difference $(p>0.05)$ in the liver and brain total thiol concentration across the groups when compared with the control, but there was a significant increase $(\mathrm{p}<0.05)$ in liver total thiol concentration in group G (acetaminophen + ampicillin/cloxacillin + co-trimoxazole) when compared to group D (acetamanophen) and a significant increase $(\mathrm{p}<0.05)$ was also observed in brain total thiol in group B and D (ampicillin/cloxacillin and acetaminophen) when compared to group C (co-trimoxazole). Furthermore, there was no significant difference $(p>0.05)$ in the serum GSH level across the groups (Table 1). However a significant decrease $(\mathrm{p}<0.05)$ in liver GSH level was observed in group $G$ (acetaminophen + ampicillin/cloxacillin + co-trimoxazole) when compared with group A, B, C and D (control, ampicillin/cloxacillin, co-trimoxazole and acetaminophen). Brain GSH level in group $\mathrm{F}$ (acetaminophen + co-trimoxazole) was also significantly elevated $(\mathrm{p}<0.05)$ when compared with the control (group A) (Table 1). 
Table 1: Total thiol and reduced glutathione levels in rats administered acetaminophen, ampicillin/cloxacillin and co-trimoxazole.

\begin{tabular}{|c|c|c|c|c|c|c|c|c|}
\hline & Groups & A & B & C & D & E & F & G \\
\hline \multirow{3}{*}{$\begin{array}{c}\text { Total thiol } \\
(m M)\end{array}$} & Serum & $0.89 \pm 0.14^{\mathrm{a}}$ & $1.52 \pm 0.09^{\mathrm{b}}$ & $0.75 \pm 0.03^{\mathrm{a}}$ & $1.75 \pm 0.08^{\mathrm{b}}$ & $1.09 \pm 0.08^{\mathrm{a}}$ & $1.00 \pm 0.18^{\mathrm{a}}$ & $1.00 \pm 0.10^{\mathrm{a}}$ \\
\cline { 2 - 8 } & Liver & $2.47 \pm 0.87^{\mathrm{a}}$ & $3.24 \pm 0.99^{\mathrm{a}}$ & $3.40 \pm 1.24^{\mathrm{a}}$ & $2.43 \pm 1.00^{\mathrm{ab}}$ & $4.37 \pm 0.50^{\mathrm{a}}$ & $3.88 \pm 1.20^{\mathrm{a}}$ & $4.97 \pm 0.42^{\mathrm{ac}}$ \\
\cline { 2 - 8 } & Brain & $1.35 \pm 0.33^{\mathrm{a}}$ & $1.84 \pm 0.18^{\mathrm{ab}}$ & $0.89 \pm 0.26^{\mathrm{ac}}$ & $1.99 \pm 0.31^{\mathrm{ab}}$ & $1.69 \pm 0.20^{\mathrm{a}}$ & $1.59 \pm 0.43^{\mathrm{a}}$ & $1.31 \pm 0.37^{\mathrm{a}}$ \\
\hline \multirow{2}{*}{$\begin{array}{c}\text { Reduced } \\
\text { glutathione } \\
(\mathrm{mM})\end{array}$} & Serum & $0.61 \pm 0.09^{\mathrm{a}}$ & $0.57 \pm 0.19^{\mathrm{a}}$ & $0.53 \pm 0.12^{\mathrm{a}}$ & $0.52 \pm 0.18^{\mathrm{a}}$ & $0.63 \pm 0.06^{\mathrm{a}}$ & $0.56 \pm 0.05^{\mathrm{a}}$ & $0.44 \pm 0.11^{\mathrm{a}}$ \\
\cline { 2 - 8 } & Liver & $0.57 \pm 0.13^{\mathrm{a}}$ & $0.57 \pm 0.10^{\mathrm{a}}$ & $0.56 \pm 0.09^{\mathrm{a}}$ & $0.52 \pm 0.05^{\mathrm{a}}$ & $0.53 \pm 0.04^{\mathrm{ab}}$ & $0.39 \pm 0.06 \mathrm{ab}$ & $0.32 \pm 0.06^{\mathrm{b}}$ \\
\cline { 2 - 8 } & Brain & $0.41 \pm 0.03^{\mathrm{a}}$ & $0.46 \pm 0.07^{\text {ade }}$ & $0.39 \pm 0.09^{\mathrm{ace}}$ & $0.40 \pm 0.05^{\mathrm{a}}$ & $0.28 \pm 0.08^{\mathrm{ac}}$ & $0.59 \pm 0.03^{\mathrm{bd}}$ & $0.55 \pm 0.04^{\mathrm{ad}}$ \\
\hline
\end{tabular}

Note: A-Control, B-Ampicillin/cloxacillin, C-Co-trimoxazole, D-Acetaminophen, E-Acetaminophen + Ampicillin/cloxacillin, F-Acetaminophen + Co-trimoxazole, G-Acetaminophen + Ampicillin/cloxacillin + Co-trimoxazole

Values are means $\pm S D, n=4$ and mean values bearing different alphabets are significantly different $(P<0.05)$ along the same row.

In Figure 1, Oxidized-Low Density Lipoprotein Cholesterol (Ox-LDL-C) concentration in the serum was elevated significantly $(\mathrm{p}<0.05)$ in group $\mathrm{F}$ and $\mathrm{G}$ ( acetaminophen + Co-trimoxazole and acetaminophen + ampicillin/cloxacillin + co-trimoxazole) when compared with the control (group A) while the elevations observed in the level of serum Ox-LDL-C in group B, C, D and E (ampicillin/ cloxacillin, co-trimoxazole, acetaminophen and acetaminophen + ampicillin/cloxacillin) were not significant ( $p>0.05)$ compared to the control (group A).

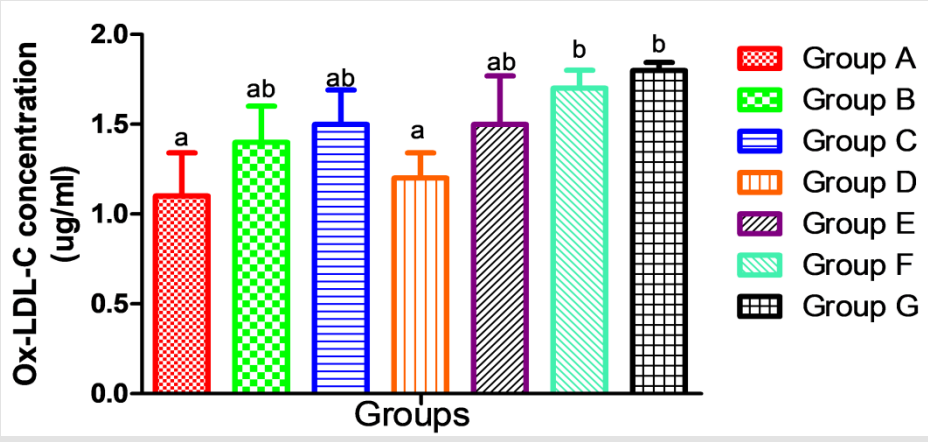

Figure 1: Serum concentration of oxidized low density-lipoprotein-cholesterol in rats following administration of acetaminophen, ampicillin/cloxacillin and co-trimoxazole.

Key: A-Control, B-Ampicillin/cloxacillin, C-Co-trimoxazole, D-Acetaminophen, E-Acetaminophen + Ampicillin/cloxacillin, F-Acetaminophen + Co-trimoxazole, G-Acetaminophen + Ampicillin/cloxacillin+Co-trimoxazole

Values are mean $\pm S D ; n=4$ and mean values bearing different alphabets are significantly different $(p<0.05)$.

\section{Inflammatory Response to Acetaminophen, Ampicillin/ Cloxacillin and Co-Trimoxazole}

The results presented in Table 2 showed no significant alterations ( $p>0.05)$ in the serum levels of Nitric Oxide (NO) and monocyte chemoattractant Protein-1 (MCP-1) across the groups ( B, C, D, E, F and G) when compared to the control (group A). Similarly, when compared to the control, there was no significant difference $(p>0.05)$ in the serum concentrations of interleukin 1- $\beta$ (Figure 2), vascular cell adhesion molecule (VCAM-1) (Figure 3), and total immunoglobulin $\mathrm{G}$ across the groups ( $\mathrm{B}, \mathrm{C}, \mathrm{D}, \mathrm{E}, \mathrm{F}$ and $\mathrm{G}$ ) when compared with the control (group A) (Figure 4). However, in Figure 3, a significant decrease $(\mathrm{p}<0.05)$ was observed in the serum level of VCAM-1 in group G (acetaminophen + ampicillin/cloxacillin + co-trimoxazole) compared to group B (ampicillin/cloxacillin).

Table 2: Serum concentrations of nitric oxide and monocyte chemoattractant Protein-1 in rats administered acetaminophen, ampicillin/cloxacillin and co-trimoxazole.

\begin{tabular}{|c|c|c|c|c|c|c|c|}
\hline Groups & A & B & C & D & E & F & G \\
\hline Nitric oxide $(\mu \mathrm{M})$ & $3.04 \pm 1.53^{\mathrm{a}}$ & $4.32 \pm 0.59^{\mathrm{a}}$ & $1.18 \pm 1.77^{\mathrm{a}}$ & $3.36 \pm .079^{\mathrm{a}}$ & $2.97 \pm 1.50^{\mathrm{a}}$ & $3.57 \pm 0.71^{\mathrm{a}}$ & $2.22 \pm 1.79^{\mathrm{a}}$ \\
\hline $\begin{array}{c}\text { Monocyte chemoattract } \\
\text { protein-1 }(\mathrm{ng} / \mathrm{ml})\end{array}$ & $156.84 \pm 26.59^{\mathrm{a}}$ & $136.77 \pm 10.20^{\mathrm{a}}$ & $160.87 \pm 7.65^{\mathrm{a}}$ & $145.36 \pm 8.26^{\mathrm{a}}$ & $141.99 \pm 12.46^{\mathrm{a}}$ & $136.86 \pm 11.64^{\mathrm{a}}$ & $167.29 \pm 32.45^{\mathrm{a}}$ \\
\hline
\end{tabular}

Note: A-Control, B-Ampicillin/cloxacillin, C-Co-trimoxazole, D-Acetaminophen, E-Acetaminophen + Ampicillin/cloxacillin, F-Acetaminophen + Co-trimoxazole, G-Acetaminophen + Ampicillin/cloxacillin + Co-trimoxazole.

Values are means $\pm \mathrm{SD}, \mathrm{n}=4$ and mean values bearing different alphabets are significantly different $(\mathrm{P}<0.05)$ along the same row. 


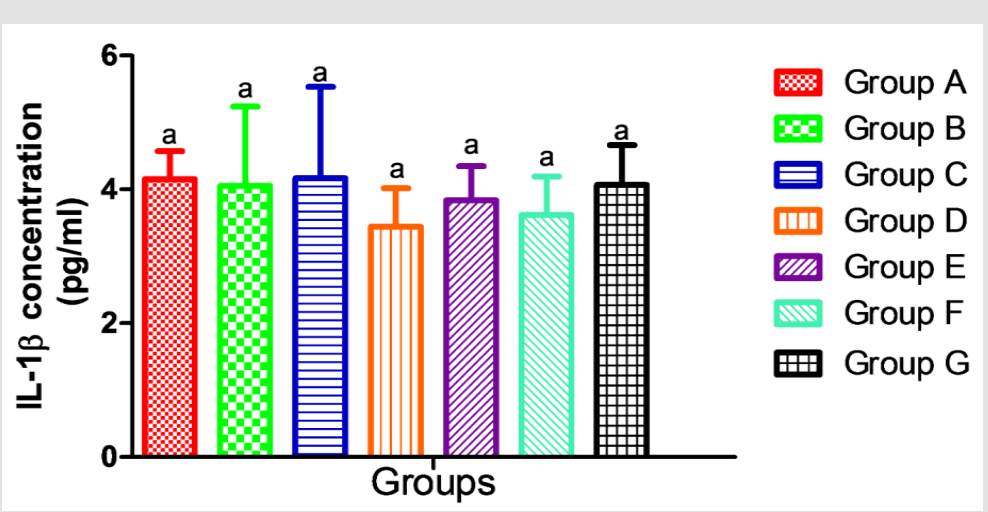

Figure 2: Concentration of Interleukin-1 $\beta$ in serum of rats administered with acetaminophen, ampicillin/cloxacillin and cotrimoxazole.

Key: A-Control, B-Ampicillin/cloxacillin, C-Co-trimoxazole, D-Acetaminophen, E-Acetaminophen + Ampicillin/cloxacillin, F-Acetaminophen + Co-trimoxazole, G-Acetaminophen + Ampicillin/cloxacillin+Co-trimoxazole

Values are means $\pm S D, n=4$ and mean values bearing different alphabets are significantly different $(P<0.05)$.

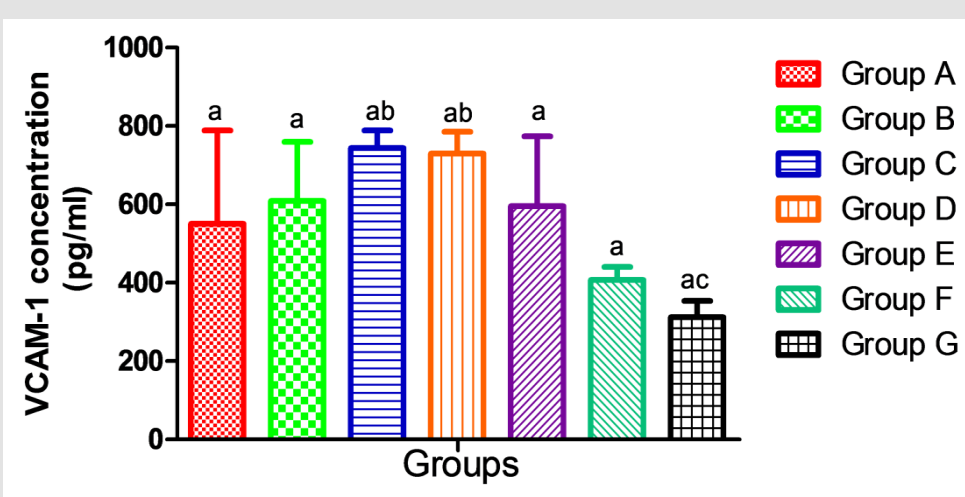

Figure 3: Serum vascular cell adhesion molecule-1 levels in rats administered acetaminophen, ampicillin/cloxacillin and cotrimoxazole.

Note: A-Control, B-Ampicillin/cloxacillin, C-Co-trimoxazole, D-Acetaminophen, E-Acetaminophen + Ampicillin/cloxacillin, F-Acetaminophen + Co-trimoxazole, G-Acetaminophen + Ampicillin/cloxacillin + Co-trimoxazole

Values are mean $\pm S D ; n=4$ and mean values bearing different alphabets are significantly different $(p<0.05)$.

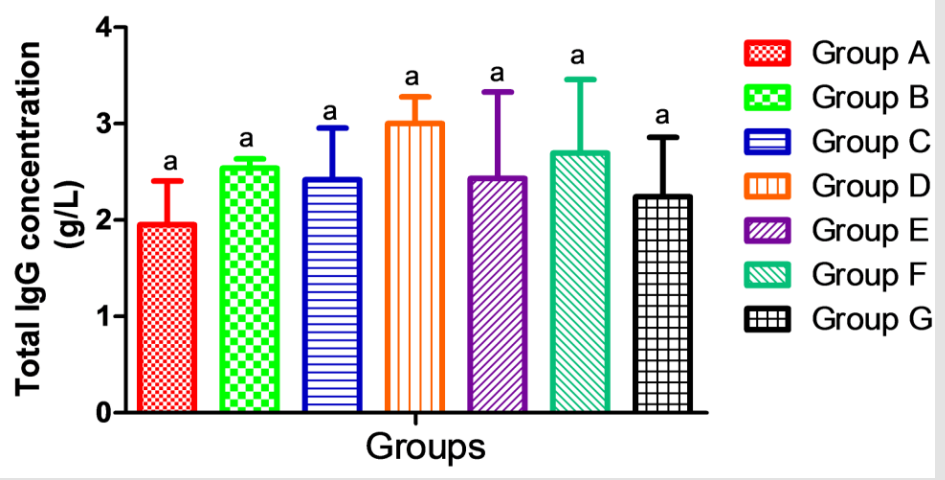

Figure 4: Total immunoglobulin G content in serum of rats administered acetaminophen, ampicillin/cloxacillin and cotrimoxazole.

Key: A-Control, B-Ampicillin/cloxacillin, C-Co-trimoxazole, D-Acetaminophen, E-Acetaminophen + Ampicillin/cloxacillin, F-Acetaminophen + Co-trimoxazole, G-Acetaminophen+Ampicillin/cloxacillin + Co-trimoxazole.

Values are means $\pm S D, n=4$ and mean values bearing different alphabets are significantly different $(P<0.05)$. 


\section{Histological Assessment of the liver and Brain Tissues}

The photomicrograph of liver section of rats in Plate 1 (A-G) showed that the hepatocytes of the control (group A) were well preserved, while various changes ranging from occlusion of the portal central vein and sinusoids (yellow arrow), loss of sinusoid lining cells and vacuolation of hepatocytes (white arrow) were observed in groups administered ampicillin/cloxacillin only (group B), co-trimoxazole only (group C), acetaminophen only (group D) and acetaminophen, ampicillin/cloxacillin and co-trimoxazole (group G). Similarly, in the group administered acetaminophen and ampicillin/cloxacillin ( group E), the sinusoids were markedly altered, population of the sinusoid lining cells was markedly reduced and the hepatocytes were undergoing degranulation and or fragmentation (yellow arrow), while the portal central vein is occluded with few fenestrations in the hepatic parenchyma of rats administered acetaminophen and co-trimoxazole (group F). In plate $2(A-G)$, neurodegeneration was observed in the cerebral cortex of rats across the groups except in the control (group A) where the cerebral cortex was preserved. In the group administered ampicillin/cloxacillin (group B), co-trimoxazole (group C), acetaminophen (group D), acetaminophen and ampicillincloxacillin (group E), acetaminophen and co-trimoxazole (group F), and acetaminophen, ampicillin/cloxacillin and co-trimoxazole (group G), the cerebral cortex of rats showed neurodegeneration depicted with red arrows (vacuolation and perinuclear spacing), black arrow (pyramidal neuron) and asterisk (blood vessel).
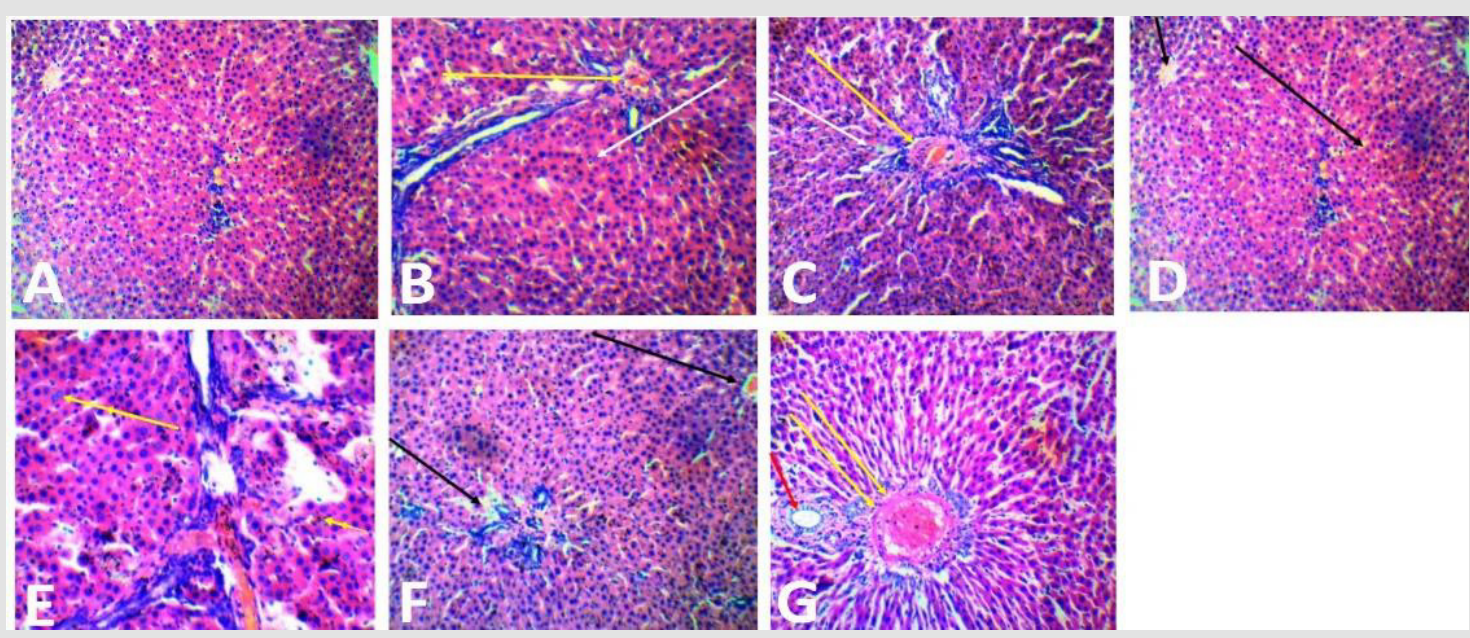

Plate 1: Photomicrograph of the liver section of rats administered with acetaminophen, ampicillin/cloxacillin, and cotrimoxazole (H\&E, 40).

Key: A-Control, B-Ampicillin/cloxacillin, C-Co-trimoxazole, D-Acetaminophen, E-Acetaminophen + Ampicillin/cloxacillin,FAcetaminophen+Co-trimoxazole, G-Acetaminophen + Ampicillin/cloxacillin + Co-trimoxazole.
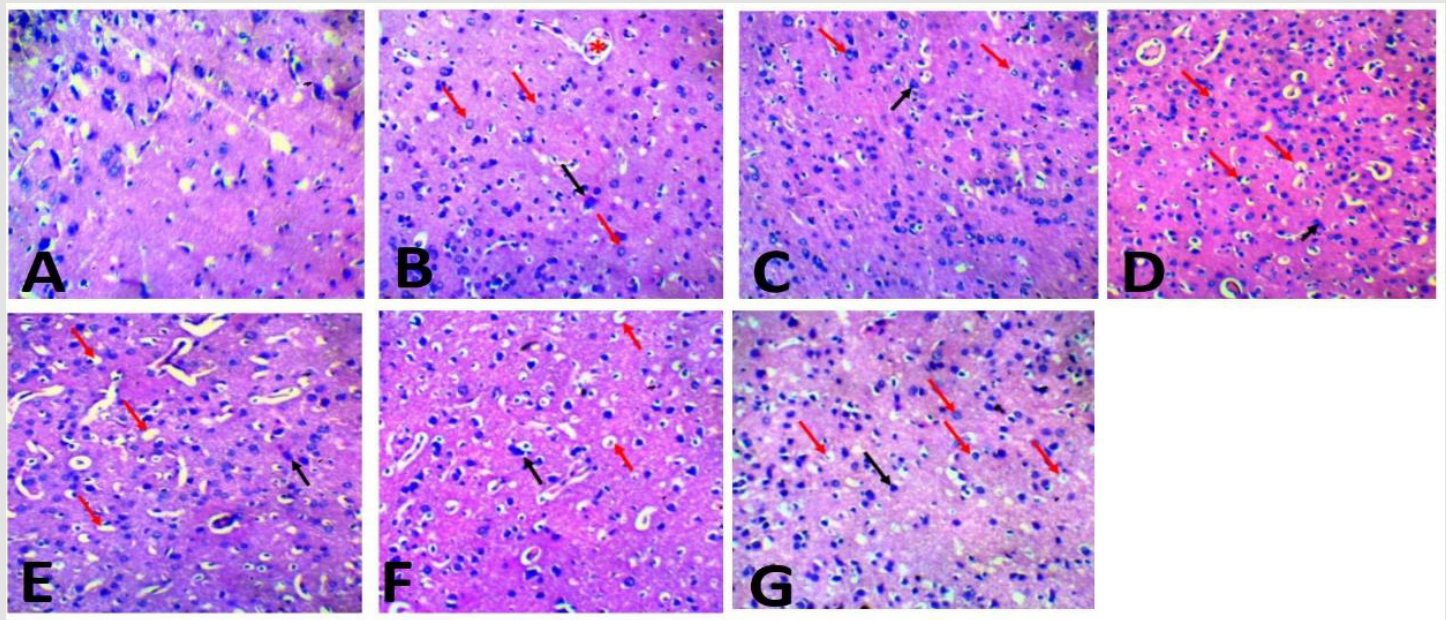

Plate 2: Photomicrograph of the cerebral cortex section of rats administered acetaminophen, ampicillin/cloxacillin, and cotrimoxazole (H\&E, x40).

Key: A-Control, B-Ampicillin/cloxacillin, C-Co-trimoxazole, D-Acetaminophen, E-Acetaminophen + Ampicillin/cloxacillin,FAcetaminophen + Co-trimoxazole, G-Acetaminophen+Ampicillin/cloxacillin + Co-trimoxazole. 


\section{Discussion}

The practice of self-medication has led to irrational/ indiscriminate use of drugs such as analgesics (acetaminophen) and antibiotics (ampicillin/cloxacillin and co-trimoxazole) especially in delevoping countries $[27,28]$. Consequently, acetaminophen is widely used alone and in combination with other drugs such as antibiotics in humans and animals [29,30]. Acetaminophen is metabolized through cytochrome P450 to produce the reactive intermediate N-acetyl-p-benzoquinonimine (NAPQI) which is normally detoxified by GSH under normal conditions [31]. However, studies have shown that detoxification of NAPQI results in depletion of GSH and the excess NAPQI causes oxidative stress [32]. Similarly, antibiotics exhibit antimicrobial effect by inducing oxidative damage in bacteria, and this is often followed by production of Reactive Oxygen Species (ROS) [15,16,33-35]. These reactive species could react with several bio-molecules including deoxyribonucleic acid, lipids, and proteins, however, proteins are prone to more risk because they often act as enzymes in cells. Significantly, all the plasma thiol groups are associated to protein and they are oxidative sensitive indexes that are employed in the defense system against ROS [36].

Depletion in levels of thiols has been noticed widely in medical disorders such as chronic renal failure and other kidney disorders, cardiovascular disorders, stroke, and other neurological disorders, diabetes mellitus, alcoholic cirrhosis and several other disorders and previous studies showed that acetaminophen significantly depleted the total thiol content of the liver tissue of rats when compared with the control group $[37,38]$. On the contrary, in this present study, an increase was observed in serum total thiol level in groups given ampicillin/cloxacillin (Group B) and acetaminophen (Group D). The total thiol assessed in this study included the oxidant (homocysteine) and antioxidant (GSH) thiol, hence, the increased serum total thiol may reflect increased concentration in the oxidant thiol group rather than the antioxidant thiol (GSH) which might be a consequence of oxidative progression in the acetaminophen administered group. This suggestion is further backed by the corresponding decrease in the concentration of the antioxidant (GSH) thiol (Table 1).

GSH is a key antioxidant defense system enzyme that is involved in detoxification of reactive oxygen species and repair resulting damage in the cell [39]. Notably, it is an essential free-radical scavenger and scavenger of NAPQI [40]. Salwe et al. in 2017 reported that the GSH amount in the liver tissue of the acetaminophenadministered rats was significantly reduced compared with that in the control group [41]. Similarly, in this present study, the depletion in liver reduced glutathione (GSH) level observed in groups administered acetaminophen and co-trimoxazole (Group F) with the lowest level observed in the group administered acetaminophen, ampicillin/cloxacillin, and co-trimoxazole (Group
G) suggests that the drugs are involved in production of reactive species and are potentially hepatotoxic with higher risk when co-administered. This opinion is strengthened by the alterations observed in the hepatocytes of rats administered separate and combined doses of acetaminophen, ampicillin/cloxacillin and cotrimoxazole (Group B-G) when compared with the hepatocytes of the control (Group A) that were well preserved (Plate 1), this may be due to exposure of the liver to large concentrations of exogenous substances and their metabolites [42].

In this study, the observed damage to the liver in rats administered acetaminophen only may be due the detoxification of the reactive intermediate of acetaminophen (NAPQI) which depletes thiol groups (Plate 1) [32]. Hepatic GSH is depleted by an overdose of acetaminophen, and NAPQI binds to cysteine residues on proteins covalently, and this leads to the formation of 3 -(cysteine-S-yl), which is detrimental to the liver $[43,44]$. Similarly, the alterations observed in the hepatocytes of rats given acetaminophen concomitantly with co-trimoxazole and ampicillin/ cloxacillin in this study may be due to an interaction between the drugs and suggests that co-trimoxazole and ampicillin/cloxacillin might be potentially hepatotoxic (Plate 1), since concomitant intake of other drugs that are potentially hepatotoxic may contribute to an increased risk of hepatotoxicity associated with the administration of acetaminophen even at a therapeutic dose [45]. This is in agreement with Sharma and Mehta [46], who stated that interaction of acetaminophen with other drugs might occur, and need caution in co-administration as this may boost the production of NAPQI and increase the risk of acetaminophen toxicity.

In addition, the brain contains a high level of polyunsaturated fatty acids and relatively low antioxidants, thereby making it vulnerable to oxidative damage [47]. The uncontrolled production of reactive oxygen species in the brain and alteration in balance of the antioxidant defense system are linked to pathological changes in neurodegenerative diseases, such as Parkinson's Disease (PD) and Alzheimer's disease (AD)[48,49]. At a therapeutic dose, acetaminophen is said to be generally safe and may protect the cerebral cortex from oxidative damage and reduce neuronal inflammation [50,51]. However, potentially fatal brain damage may be caused by acute overdose of acetaminophen, and even after a therapeutic dose in few individuals, which may be due to the presence of subclinical risk factors such as deficiency of glutathione, 'fast-metabolizer' status, or both [46]. In previous studies, Nencini et al. reported that overdose acetaminophen reduced GSH levels and induced oxidative stress in the brain. In contrast [52], in this present study an increase was observed in brain GSH levels in rats administered acetaminophen and co-trimoxazole (Group F) (Table 1 ), this might be a consequence of an oxidative progression where the degree of oxidation has not exceeded the antioxidant capacity. However, when the progression of oxidation overwhelms the 
capacity of the antioxidant defense system, antioxidant molecules such as GSH levels might be reduced [53]. Similarly, the trend observed in the brain levels of total thiol across the groups showed that the drugs had no effect on total thiol levels at the administered doses (Table 1).

Regardless of the pattern observed in the brain levels of total thiol and GSH, the histological assessment of the brain showed neurodegeneration in the cerebral cortex of rats given acetaminophen only as well as in rats given the combination of acetaminophen with ampicillin/cloxacillin and co-trimoxazole (Plate 2). The ability to effectively access cellular damage through histological assessment depends on several factors such as the number of injured tissues, size of the sample and tissue sectioning during histopathological preparations [54]. Thus, the inconsistency between the brain histological examinations, total thiol and GSH levels in this study might be due to these factors. However, the findings in this study is similar to that of reports from previous studies in which several degenerative changes occur in the brain cells in response to acetaminophen treatment and acetaminophen had a detrimental effect on cortical neurons both in vivo and in vitro $[55,56]$. This suggests that acetaminophen as well as ampicillin/cloxacillin and co-trimoxazole might be implicated in the histopathological changes associated with neurodegenerative conditions. Neurodegeneration may result in a clinical syndrome referred to as dementia [57], which characterize a set of symptoms such as memory loss, mood changes, and difficulty with communication and reasoning.

Ultimately, the trend observed in the levels of total thiol and GSH concomitant administration of ampicillin/cloxacillin, cotrimoxazole and acetaminophen suggested an oxidative stress state (Table 1). This is supported by many studies in which it has been observed that drugs such as anti-inflammatory and analgesic could induce oxidative stress that is accompanied by increased cellular oxidants [17], lipid peroxidation and depletion of antioxidants. Moreover, antibiotics exerts its therapeutic effect in bacteria by inducing a oxidative damage that results in formation of Reactive Oxygen Species (ROS) and alteration to DNA, protein, and lipid intergrity which results in cell death $[15,16,33,34]$.

Furthermore, various clinical studies proved that low density lipoprotein is prone to oxidative damage. The Oxidized Low Density Lipoprotein Cholesterol (Ox-LDL-C) formed as a result of this oxidation has much pathobiological significance [58]. Oxidation of Low-Density Lipoprotein Cholesterol (LDL-C), and the following engulfment by macrophages in the vascular wall are significant steps in atherosclerosis development [59]. A small part of the OxLDL-C particles escapes uptake by macrophages and returns to the bloodstream or may leak from atherosclerotic plaques. Thus, measuring circulating levels of Ox-LDL-C may contribute to the estimation of Cardiovascular Disease (CVD) risk. The increase in the Ox-LDL-C observed in the group given acetaminophen and ampicillin/cloxacillin (Group E), acetaminophen and co-trimoxazole (Group F), and the mixture of acetaminophen, ampicillin/cloxacillin, and co-trimoxazole (Group G) might therefore be as a result of the oxidative reaction reported earlier in the result of total thiol and GSH and may suggest cardiovascular risks (Figure 1).

A link between oxidative stress and pro-inflammatory cytokine production has been identified [60]. Conversely, as reported in a study conducted by Wu [61], Ox-LDL-C did not change after adjustment of any inflammatory markers; therefore, the association of $\mathrm{Ox}$-LDL-C was not explained by inflammation. For instance, pro-

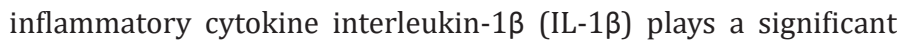
role in the signal transduction of immune stimuli from the peripheral to the central nervous system, and has been considered to be a significant mediator of the immune-induced stress hormone release. In this study, no significant change was observed in the levels of interleukin- $1 \beta$ (Figure 2), this is in agreement with the trend observed by Hadzi-Petrushev et al. [62], who stated that the acetaminophen-induced analgesic toxicity was not associated with elevated level of interleukin- $1 \beta$, and the trend observed in concentrations of MCP-1, nitric oxide, and VCAM-1 also agrees with that of interleukin-1 $\beta$ (Table 2) (Figure 3). Although, the trends in Ox-LDL-C indicated otherwise, however, pattern observed in the immunological markers suggests that there was no active progression of inflammatory responses that are vascular related at the administered doses of the drugs.

Paradoxically, the oxidation of low density LipoproteinCholesterol (LDL-C) is the hallmark of all vascular related diseases in which the uptake by macrophages initiate the release of other chemokines (NO, MCP-1) and cytokines (Il-1 $\beta$ ). However, significant increase of chemokines and cytokines are observable as atherosclerotic plaques starts to form [63-65]. Thus, progression of vascular related disorder is known as a very salient process which is only evident after many accumulation events. In this present study, the increase in Ox-LDL-C that was accompanied with no significant change in inflammatory markers suggests that the duration and level of Ox-LDL-C was not sufficient to increase the levels of the inflammatory markers.

\section{Conclusion}

In conclusion, this study showed that the concomitant use of acetaminophen, ampicillin/cloxacillin and co-trimoxazole could induce oxidative stress that could be weakly related to vascular related disorders. Also, the prolonged administration of these drugs might be potentially hepatotoxic and have neurodegenerative effect.

\section{Ethical Approval and Consent to Participate}

This study was carried out in accordance with ethical laws on animal handling. 


\section{Funding}

We did not receive any grant for the conduct of the study.

\section{Authors' Contributions}

EBO conceived, designed and supervised the study, and drafted the manuscript. OJB provided the materials used in the study, AJO collated literatures and performed the experimental procedures and did the statistical analyses AAS co-supervised the study and read the final manuscript. All authors financed of the study. All authors read and approved the final manuscript with the order of author's names.

\section{Acknowledgements}

We acknowledge the immense efforts and laboratory assistance of Dr. R. A. Ajani (LAUTECH) and the technical inputs of Messr Olabanji Debo of Bridge Scientifik Limited.

\section{References}

1. Ibrahim HA, Mahmud S, Abubakar A, Harazimi A, Abdukadir S (2016) Effects of drug abuse among youths and its impact on learning. International Organization of Scietific Research (IOSR) J Pharm Biol Sci 11: 14-17.

2. Bennadi D (2013) Self-medication: a current challenge. J Basic Clin Pharm 5: 19-23.

3. Hernandez-Juyol M, Job-Quesada JR (2002) Dentistry and selfmedication: a current challenge. Med Oral 7: 344-347.

4. Martins AP, Miranda AC, Mendes Z, Soares MA, Ferreira P, et al. (2002) Self-medication in a Portuguese urban population: a prevalence study. Pharmacoepidemiol Drug Safety 11: 409-414.

5. Alam N, Saffoon N, Uddin R (2015) Self-medication among medical and pharmacy students in Bangladesh. Biomed Central Res Notes 8: 763.

6. Shehnaz SI, Agarwal KA, Khan N (2014) A systematic review of selfmedication practices among adolescents. J Adolesc Health 55: 467-483.

7. Koushede V, Hansen EH, Andersen A, Holstein BE (2012) Young adults medicine use for headache: the combined effect of socioeconomic position and perceived stress, and the contribution of sense of coherence. Res Social Adm Pharm 8(6): 533-541.

8. Albsoul-Younes AM, Jabateh SK, Abdel-Hafiz SM, Al-Safi SA (2004) Awareness and frequency of potential side effects on nonsteroidal antiinflammatory drugs among the Jordanian patient population. Saudi Med J 25(7): 907-911

9. Józwiak-BebeniSta M, Nowak JZ (2014) Acetaminophen: mechanism of action, applications and safety concern. Acta Pol Pharm 71: 11-23.

10. Siemionow K, Teul J, Dragowski P, Palka J, Miltyk W (2016) New potential biomarkers of acetaminophen-induced hepatotoxicity. Adv Med Sci 61: 325-330.

11. Skliros E, Merkouris P, Papazafiropoulou A (2010) Self-medication with antibiotics in rural population in Greece: A cross-sectional multicenter study. BMC Fam Pract 11: 58.

12. Sneader W (2008) Drug discovery: a history. Chichester. John Wiley and Sons. pp. 321

13. Ashnagar A, Gharib N (2007) Analysis of three penicillins (Ampicillin, Cloxacillin and Amoxillin) of several Iranian Pharmaceutical companies by HPLC. E-J Chem 4(4): 536-545.
14. Oyetunde 00, Olugbake OA, Famudehin KF (2010) Evaluation of use of antibiotic without prescription among young adults. Afr J Pharm Pharmacol 4(10): 760-762.

15. Kohanski MA, Dwyer DJ, Hayete B, Lawrence CA, Collins JJ (2007) A common mechanism of cellular death induced by bactericidal antibiotics. Cell 130: 797-810.

16. Wang X, Zhao X (2009) Contribution of oxidative damage to antimicrobial lethality. Antimicrob Agents Chemother 53: 1395-1402.

17. Sha L, Hor-Yue T, Ning W, Zhang-Jin Z, Lixing L, et al. (2015) The role of oxidative stress and antioxidants in liver disease. Int J Mol Sci 16: $26087-$ 26124.

18. Morgan MJ, Liu ZG (2011) Crosstalk of reactive oxygen species and NFkappaB signaling. Cell Res 21: 103-115.

19. Iqbal Z, Khan A, Naz A, Khan AJ, Khan SG (2009) Pharmacokinetic interaction of ciprofloxacin and diclofenac: a single dose, two period crossover study in healthy adults volunteers. Clin Drug Investig 29(3): 1.

20. Yoon E, Babar A, Choudhary M, Kutner M, Pyrsopoulos N (2016) Acetaminophen-induced hepatotoxicity: a comphrensive update. J Clin Transl Hepatol 28: 131-142.

21. Ayogu E, Ugwowo O, Amorha KC, Okonta JM (2016) Evaluation of ciprofloxacin effect on the antimalaria activity of some anti-malaria drugs in plasmodium Berghei infected mice. Int J Pharm Sci Res 7(5):1896-1903.

22. Reagan-Shaw S, Nihal M, Ahmad N (2008) Dose translation from animal to human studies revisited. FASEB J 22(3): 659-661.

23. Sedlak J, Lindsay RH (1968) Estimation of total, protein-boud and nonprotein sulfhyhydryl groups in tissue with Ellman's reagent. Anal Biochem 25(1): 192-205.

24. Ellman GL (1959) Tissue sulfyhydrl groups. Arch Biochem Biophys 82 70-77.

25. Schmidt HH (1995) Non-enzymatic colorimetric assay for nitric oxide. Biochem 2: 22-23.

26. Avwioro OG (2001) Histochemistry and tissue pathology: principle and techniques. Claverianum J 15(8): 45-77.

27. Afolabi AO (2000) Factors influencing the pattern of self medication in an adult Nigerian population. Ann Afr Med 7(3): 120-127.

28. Shehnaz SI, Agarwal KA, Khan N (2014) A systematic review of selfmedication practices among adolescents. J Adolesc Health 55: 467-483.

29. Dargan P, Jones A (2002) Paracetamol: balancing risk against benefit. QJM Int J Med 95(12): 831-832.

30. Sheen CL, Dillon JF, Bateman DN, Simpson KJ, Macdonald TM (2002) Paracetamol toxicity: Epidemiology, prevention and costs to the healthcare system. QJM Int J Med 95(9): 609-619.

31. Tezcan AH, Ozturk O, Ustebay S, Adali Y, Yagmurdur H (2018) The beneficial effects of ozone therapy in acetaminophen-induced hepatotoxicity in mice. Pharmacol Rep 70: 340-345.

32. Bessems JG, Vermeulen NP (2001) Paracetamol (acetaminophen)induced toxicity: molecular and biochemical mechanisms, analogues and protective approaches. Crit Rev Toxicol 31(1):55-138.

33. Kohanski MA, Dwyer DJ, Wierzbowski J, Cottarel G, Collins JJ (2008) Mistranslation of membrane proteins and two-component system activation trigger antibiotic-mediated cell death. Cell 135: 679-690.

34. Foti JJ, Devadoss B, Winkler JA, Collins IJ, Walker GC (2012) Oxidation of the guanine nucleotide pool underlies cell death by bactericidal antibiotics. Science 336: 315-319. 
35. Shatalin K, Shatalina E, Mironov A, Nudler E (2011) $\mathrm{H}_{2} \mathrm{~S}$ : a universal defense against antibiotics in bacteria. Science 334(658): 986-990.

36. Terziogl D, Uslu L, Simsek G, Atukeren P, Erman H, et al. (2017) The effects of hyperbaric oxygen treatment on total antioxidant capacity and prolidase activity after bile duct ligation in rats. J Invest Surg 30: 376 382.

37. Mungli P, Mahesh SS, Prasiddha T, Naureen A (2009) Total thiols: biomedical Importance and their alteration in various diseases. Online ] Health Alllied Sci 8(2).

38. Kabirifar R, Safari F, Karimollah A, Moradi A, Eskandari-nasab E (2017) Quercetin protects liver injury induced by bile duct ligation via attenuation of Rac1 and NADPH oxidase1 expression in rats. Hepatobiliary Pancreat Dis Int 16: 88-95.

39. Whidden MA, Kirichenko N, Halici Z, Erdos B, Foster TC, et al. (2011) Lifelong caloric restriction prevents age-induced oxidative stress in the sympathoadrenal system of 29 Fischer 344 x Brown Norway rats. Biochem Biophys Res Commun 408(3): 454-458.

40. Yayla M, Halici Z, Unal B, Bayir Y, Akpinar E, Gocer F. 2014. Protective effect of et-1 receptor antagonist bosentan on paracetamol induced acute liver toxicity in rats. Eur J Pharmacol. 726:87-95.

41. Hajhashemi V, Ghannadi A, Sedighifar S (2007) Analgesic and antiinflammatory properties of the hydroalcoholic, polyphenolic and boiled extracts of Stachyslavandulifolia. Res Pharm Sci 1: 92-98.

42. Yaacov B (2000) The liver: a large endocrine gland. J Hepatol 32(8): 505507.

43. Ishida Y, Kondo T, Tsuneyama K, Lu P, Takayasu T, et al. (2004) The pathogenic roles of tumor necrosis factor receptor p55 in acetaminophen-induced liver injury in mice. J Leukoc Biol 75: 59-67.

44. Stannard C, Booth S (2004) Pain. Elsevier-Churchill Livingstone, London, UK.

45. Karwoski CB (2002) Briefing document: acetaminophen containing products and hepatotoxicity. Division of Drug Risk Evaluation.

46. Sharma CV, Mehta V (2014) Acetaminophen: mechanism and updates. Br J Anaesth 14: 4.

47. Butterfield DA, Castegna A, Lauder CM, Drake J (2002) Evidence that amyloid beta-peptide-induced lipid peroxidation and its sequelae in Alzhemimer's disease brain contribute to neuronal death. Neurobiol Aging 23: 655-664

48. Von Campenhausen S, Bornschein B, Wick R, Botzel K, Sampaio C, et al. (2005) Prevalence and incidence of Parkinson's disease in Europe. Eur Neuropsychopharmacol 15: 473-490.

49. Halliwell B (2006) Oxidative stress and neurodegenaration: where are we now? J Neurochem 97(6): 1634-1658.

50. Bisaglia M, Venezia V, Piccioli P, Stanzione S, Porcile C, et al. (2002) Acetaminophen protects hippocamal neurons and PC12 cultures from

ISSN: 2574-1241

DOI: 10.26717/BJSTR.2021.38.006135

Oyewo EB. Biomed J Sci \& Tech Res

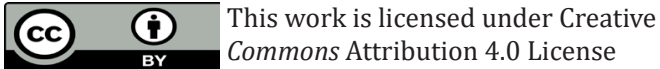

Submission Link: https://biomedres.us/submit-manuscript.php amyloid beta-peptides inducedoxidative stress and reduces NF-kappaB activation. Neurochem Int 41(1): 43-54.

51. Tripathy D, Grammas P (2009) Acetaminophen inihibits neuronal inflammation and protects neurons from oxidative stress. J Neuroinflammation 6: 10

52. Nencini C, Giorgi G Micheli L (2007) Protective effect of silymaria on oxidative stress. Phytomedcine 14: 129-135.

53. Xu M, Rui D, Yan Y, Xu S, Niu Q, et al. (2017) Oxidative damage induced by arsenic in mice/rats: a systemic review and meta-analysis. Biol Trace Elem Res 176: 154-175.

54. Khayyat AN (2016) Contribution of new thiol antioxidant in the treatment of acetaminophen toxicity. Doctoral Dissertations. pp. 2447.

55. Posadas I, Santos P, Blanco A, Munoz-Fernadez M, Cena V (2010) Acetaminophen induce apoptosis in rat cortical neurons. PloS One 5(12): e 15360

56. Essawy AE, Alkhuriji AF, Soffar AA (2017) Paracetamol Overdose induces physiological and pathological aberrations in rat brain. J Applied Pharm Sci 7(9): 185-190.

57. Wittchen HU, Jacobi F, Rehm J, Gustavsson A, Svensson M, et al. (2011) The size and burden of mentaldisorders and other disorders of the brain in Europe 2010. Eur Neuropsychopharmacol 21: 655-679.

58. Ehara S, Ueda M, Naruko T, Haze Itoh A, Otsuka M, et al. (2001) Elevated levels of oxidized low density lipoprotein show a positive relationship with the severity of acute coronary syndromes. Circulation 103(52): 1955-1960.

59. Lusis AJ (2000) Atherosclerosis. Nature 407: 233-241.

60. Ghosh J, Das J, Manna P, Sil PC (2010) Acetaminophen induced renal injury via oxidative stress amd TNF-alpha production. Therapeutic potential of arjunolic acid. Toxicology 268: 8-18.

61. Wu T, Willett WC, Rifai N, Shai I, Manson JE, et al. (2006) Is plasma oxidized low density lipoprotein, measured with widely used Antibody 4E6, an independent predictor of coronary heart disease among U.S men and women? J Am Coll Cadiol 48(5): 973-979.

62. Hadzi-Petrushev N, Mitrov D, Kostovski V, Mladenov M (2017) The impact of vitamin $\mathrm{C}$ on the relationship among inflammation, lipid peroxidation and platelet activation during anagelsic nephropathy in rats. J Basic Clin Physiol Pharmacol 28(5): 473-481.

63. Li, Cybulsky MI, Gimbrone MA, Libby P (1993) Arteriosclerosis thrombosis 13: 197-203.

64. Libby P, Ridker PM, Maseri A (2002) Inflammation and atherosclerosis. Circulation 105: 1135-1142.

65.Zipes DP, Libby P, Bonow RO, Braunwald E (2005) Braunwald's heart disease. A textbook of cardiovascular medicine $\left(7^{\text {th }}\right.$ edn). Pennsylvania, Elsevier Saunders, USA.

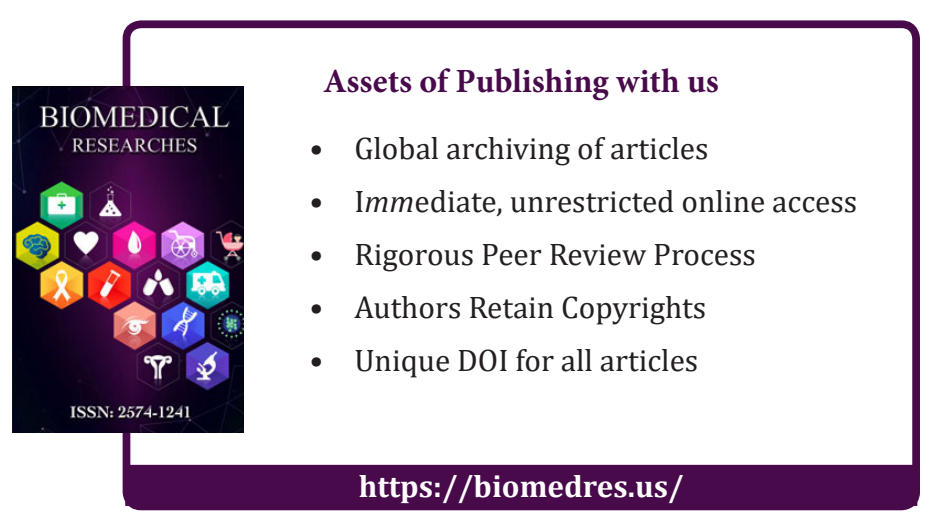

